\title{
Undifferentiated connective tissue disease: a seven-center cross-sectional study of 184 patients
}

\author{
C. C. Vaz • M. Couto • D. Medeiros • L. Miranda • \\ J. Costa - P. Nero - R. Barros - M. J. Santos - E. Sousa • \\ A. Barcelos • L. Inês
}

Received: 7 January 2009/Revised: 25 March 2009 /Accepted: 26 March 2009

(C) Clinical Rheumatology 2009

\begin{abstract}
The purpose of this study was to characterize the clinical and serological features of a large cohort of patients with antinuclear antibody (ANA) positive undifferentiated connective tissue disease (UCTD). Consecutive patients
\end{abstract}

C. C. Vaz $(\bowtie) \cdot$ M. Couto $\cdot$ L. Inês

Department of Rheumatology, Coimbra University Hospital,

Praceta Prof. Mota Pinto,

3000-075 Coimbra, Portugal

e-mail: claudiacvaz@gmail.com

M. Couto

Institute of Physiology, Faculty of Medicine, Coimbra University,

Coimbra, Portugal

D. Medeiros $\cdot$ L. Miranda

Portuguese Institute of Rheumatology,

Lisbon, Portugal

J. Costa

Department of Rheumatology, Alto Minho Hospital Centre,

Ponte de Lima, Portugal

P. Nero $\cdot$ R. Barros

Department of Rheumatology, Egas Moniz Hospital,

Lisbon, Portugal

\section{J. Santos}

Department of Rheumatology, Garcia de Orta Hospital,

Almada, Portugal

E. Sousa

Department of Rheumatology, Santa Maria Hospital,

Lisbon, Portugal

\author{
A. Barcelos \\ Rheumatology Unit, Infante D. Pedro Hospital, \\ Aveiro, Portugal \\ L. Inês \\ Faculty of Health Sciences, University of Beira Interior, \\ Covilhã, Portugal
}

with UCTD, followed up at the Rheumatology Clinic of the participating centers, were included. Data from these patients were obtained by clinical evaluation and chart review. All patients were diagnosed as having UCTD on basis of the following criteria: positive ANA plus at least one clinical feature of connective tissue disease, but not fulfilling classification criteria for any differentiated connective tissue disease. One hundred eighty-four patients were studied (female patients - $94.5 \%$; mean age at time of evaluation - 47 years). The most prevalent manifestations were arthralgia (66\%), arthritis (32\%), Raynaud's phenomenon (30\%), sicca symptoms (30\%), and leukopenia (19\%). The prevalence of ANA was $100 \%$, anti-SSA $20 \%$, antidsDNA $14 \%$, and anti-SSB 7\%. Patients with anti-dsDNA/ anti-Sm, anticentromere/anti-Scl70, or anti-SSA/anti-SSB antibodies more frequently presented a set of manifestations close to systemic lupus erythematosus (SLE), systemic sclerosis, or Sjögren syndrome, respectively. We analyze a large cohort of UCTD. Seventy-two percent of these UCTD patients present lupus-, scleroderma-, or Sjögren-like features but do not fulfill classification criteria and mostly present a mild disease.

Keywords Classification criteria - Connective tissue diseases · Systemic lupus erythematosus .

Undifferentiated connective tissue diseases

\section{Introduction}

Many patients present at Rheumatology Clinic because of signs, symptoms, and laboratory abnormalities suggesting systemic rheumatic disease, but do not meet classification criteria of any specific connective tissue disease (CTD) or any other well-defined disease that might justify these 
features. These syndromes are currently classified as undifferentiated connective tissue disease (UCTD).

Consensual classification criteria for UCTD have not been attained. However, Mosca et al. [1] proposed preliminary classification criteria for UCTD, comprising (1) patients presenting signs and symptoms suggestive of a CTD, but not fulfilling the criteria for any of the defined CTDs, and (2) presence of antinuclear antibodies determined on two different occasions. Additionally, those with disease duration less than 3 years would be defined as having an early UCTD.

There is a need for sensitive, specific, and consensual classification set, enabling the exclusion of patients with no autoimmune disturbance and ensuring comparability between studies. There is also a need for prognosis estimation criteria, including risk of the disease's progression and of the development of severe clinical manifestations.

There is still scarcely any data regarding the possibility to prevent progression of UCTD to a specific CTD with immunomodulatory drugs. Interestingly, a recent prospective study of $25(\mathrm{OH})$ D3 serum levels in patients with UCTD suggests that vitamin D deficiency in UCTD patients may increase the rate of progression into welldefined CTDs [2]. In SLE, early intervention with hydroxychloroquine has been postulated to delay or prevent the development of more serious sequelae. In another study, UCTD patients treated with hydroxychloroquine who subsequently progressed to systemic lupus erythematosus had a longer lag time between the onset of the first clinical symptom and SLE classification and also had a lower rate of autoantibody accumulation and a decreased number of autoantibody specificities at and after diagnosis [3].

Our study presents the characterization of the clinical and serological profile of a large cohort of UCTD patients followed in seven Rheumatology Units.

\section{Materials and methods}

Seven Rheumatology Outpatient Clinics participated in this study, which represented $58 \%$ of all Portuguese centers and covering a representative sample of the Portuguese population. The inclusion criteria were consecutive patients seen at the participating clinics and fulfilling the UCTD classification criteria proposed by Mosca et al. [1], regardless of the disease's duration. Exclusion criteria were patients fulfilling classification criteria for any differentiated CTD or any well-defined disease that justifies the recorded clinical and analytical changes, including rheumatoid arthritis, systemic lupus erythematosus, systemic sclerosis, Sjögren syndrome, psoriatic arthritis, seronegative spondyloarthritis, juvenile idiopathic arthritis, antiphospholipid syndrome, systemic vasculitis, or fibromyalgia. Clin- ical and laboratorial data were obtained by clinical evaluation at time of visit and retrospective analysis of patients with UCTD diagnosis clinical charts followed up between 1989 and 2007. Antinuclear antibody (ANA) testing was requested by the attending rheumatologist if there was a clinical suspicion of CTD. Sera were sent to the respective center immunology laboratory, where ANA was determined by indirect immunofluorescence on Hep-2 cells. Titers of 1:160 or greater were considered positive. In ANA positive sera, further testing was done. Anti-dsDNA was evaluated by Farr radioimmunoassay or enzyme-linked immune-absorbent assay (ELISA), depending on the laboratory. Anti-RNP, antiSm, anti-SSA, anti-SSB, anti-Jo1, anti-Scl70, and anticardiolipin were analyzed in all patients with ELISA. Lupic anticoagulant was detected by means of two triage tests using Dilute Russell Viper Venom time. Determination of C3 and C4 was assessed by nephelometry. A protocol chart (Table 1) was elaborated to record each patient's data. After screening of inclusion and exclusion criteria, data were introduced and analyzed in Excel $^{\circledR}$. Thirteen patients were excluded at this point, 11 because they presented negative ANA and two because they had an established primary antiphospholipid syndrome. Qui-Square tests were used to analyze the differences between groups.

\section{Results}

A total of 184 patients ( $94.5 \%$ female patients) was included, mean age 47 years (16-78 years). Mean followup after diagnosis, 3.04 years (up to 18 years), presented an early UCTD $53 \%$ of the patients.

The most frequent clinical-laboratorial manifestations, with prevalence $>10 \%$, were arthralgia (66\%), arthritis (32\%), sicca syndrome (30\%), Raynaud's phenomenon $(30 \%)$, leukopenia (19\%), photosensitivity $(17 \%)$, anemia $(15 \%)$, oral ulcerations $(14 \%)$, alopecia $(13 \%)$, and arterial hypertension $(13 \%)$. Other manifestations were rare, including major organ involvement (Table 2). Each patient presented a mean of three clinical manifestations.

Concerning autoimmune profile, $100 \%$ presented positive ANA, as required by inclusion criteria. The majority of patients presented positive ANA with no detectable antigen specificity ( $61 \%$ of patients), $26 \%$ had autoantibodies against a single nuclear antigen, $11 \%$ had two antigen specificities and $3 \%$ had three. Anti-SS-A (20\%), antidsDNA (14\%), and anti-SS-B (7\%) were the most prevalent autoantibodies. The prevalence of anti-RNP was $6 \%$, anticentromere was $4 \%$, anti-histones was $4 \%$, anti-Sm was $2 \%$, and anti-Scl-70 was $1 \%$. Some patients $(10 \%)$ also presented positive anticardiolipin antibodies.

In $17 \%$ of patients, serum levels of $\mathrm{C} 3$ and/or $\mathrm{C} 4$ were low. Of these, $18 \%$ presented anti-dsDNA, $30 \%$ anti-SSA, 
Table 1 Protocol chart

\begin{tabular}{ll}
\hline Signs and symptoms & Serological manifestations \\
\hline Arthralgia/arthritis & ANA \\
Raynaud's phenomenon & Pattern: homogeneous/anticentromere/antinuclear \\
Xerophthalmia/xerostomia & Titers \\
Leukopenia/anemia/thrombocytopenia & Anti-ds-DNA/Anti-ss-DNA/anti-Sm/antihistones \\
Alopecia & Anti-SSA/anti-SSB/anti-RN/anti-Jo-1/anti-Scl \\
& 70/anticentromere \\
Photosensitivity & Ac antiphospholipid/anticardiolipin/lupic anticoagulant \\
Serositis & Decreased C3/Decreased C4 \\
Malar rash/discoid rash & Therapeutic: NSAIDs/corticosteroids/antipaludics/ \\
& azathioprine/others \\
Oral ulcers & \\
Serositis & \\
Pulmonary fibrosis & \\
Arterial hypertension/pulmonary arterial & \\
hypertension & \\
Sclerodactyly & \\
Disphagia & \\
Telangiectasia & \\
\hline
\end{tabular}

$11 \%$ anti-SSB, 5\% anticentromere, 5\% anti-RNP, 2\% antihistones, and $30 \%$ no detectable antigen specificity of ANA.

Many patients with UCTD included in the cohort presented features similar to SLE, but did not fulfill, up to the point of assessment, this disease's classification criteria $[4,5]$. Seventy-four patients $(40 \%$ of the cohort) met three classification criteria for SLE and thus presented a lupuslike disease. Clinical and serological characterization of this UCTD subgroup is presented in Table 3 and compared with the rest of the cohort. The lupus-like subgroup presented

Table 2 Rare clinical and laboratorial manifestations ( $<10 \%$ prevalence)

\begin{tabular}{lc}
\hline & Patients (\%) \\
\hline Thrombocytopenia & 9 \\
Malar rash & 8 \\
Sclerodactylia & 2 \\
Telangiectasia & 2 \\
Serositis & 2 \\
Livedo reticularis & 2 \\
Erythema nodoso & 2 \\
Dysphagia & 1 \\
Discoid rash & 1 \\
Pulmonary fibrosis & 1 \\
Proteinuria & 1 \\
Leukocytoclastic vasculitis & 1 \\
Uveitis & 0.5 \\
Autoimmune thyroiditis & 0.5 \\
Pulmonary hypertension & 0.5 \\
Myositis & 0.5 \\
\hline
\end{tabular}

higher frequency of arthritis, cytopenias, oral ulcers, photosensitivity, and anti-dsDNA antibody.

The presence of anti-dsDNA was associated with decreased levels of C3 $(p=0.024)$ and C4 $(p=0.007)$.

Table 3 UCTD clinical and serological manifestations/lupus-like subgroup

\begin{tabular}{|c|c|c|c|}
\hline & $\begin{array}{l}\text { Non lupus-like } \\
\text { subgroup; } N=110(\%)\end{array}$ & $\begin{array}{l}\text { Lupus-like } \\
\text { subgroup; } \\
N=74(\%)\end{array}$ & $p$ \\
\hline Arthralgia & 58 & 54 & 0.047 \\
\hline Raynaud & 35 & 22 & n.s. \\
\hline Arthritis & 24 & 49 & $<0.0001$ \\
\hline Leukopenia & 12 & 28 & 0.006 \\
\hline Anemia & 8 & 24 & 0.006 \\
\hline Sicca syndrome & 35 & 28 & n.s. \\
\hline Photosensitivity & 12 & 25 & 0.006 \\
\hline Serositis & 0 & 4 & n.s. \\
\hline Malar rash & 5 & 13 & n.s. \\
\hline Oral ulcers & 8 & 22 & 0.003 \\
\hline Thrombocytopenia & 5 & 14 & 0.035 \\
\hline Positive ANA & 100 & 100 & - \\
\hline Anti-dsDNA & 4 & 29 & $<0.0001$ \\
\hline Anti-SSA & 24 & 21 & n.s. \\
\hline Anti-SSB & 10 & 4 & n.s. \\
\hline Anti-Sm & 1 & 4 & n.s \\
\hline Anti-RNP & 5 & 4 & n.s. \\
\hline Anti-Scl 70 & 1 & 0 & n.s. \\
\hline Anticentromere & 5 & 3 & n.s \\
\hline Antihistones & 2 & 5 & n.s. \\
\hline
\end{tabular}


Anticardiolipine antibodies related to thrombocytopenia $(p=0.014)$.

Only $4.8 \%$ of patients presented anticentromere antibodies or anti-Scl70. The subgroup of patients with either of these antibodies presented systemic sclerosis-like clinical changes, with higher prevalence of Raynaud's phenomenon and telangiectasia and lower frequency of arthritis, as compared with the rest of the UCTD cohort, but not fulfilling classification criteria for systemic sclerosis (SSc) [6-8] (Table 4). Patients with anti-SSA and/or anti-SSB antibodies (27\% of the cohort; Table 5) presented a higher prevalence of leukopenia and sicca syndrome but not of other clinical features suggestive of Sjögren-syndrome (SS) [9, 10].

The remaining patients ( $44 \%$ of the cohort) showed mild clinical-laboratorial changes, characterized mostly by arthralgia (57\%), Raynaud (32\%), sicca symptoms (25\%), arthritis (24\%), photosensitivity (10\%), and leukopenia (9\%). In this subgroup, prevalence of anti-RNP was $7 \%$, anti-dsDNA was $2 \%$, antihistones were $2 \%$, anti-SSA was $1 \%$, and anti-Sm $1 \%$.

Most patients with UCTD belonging to this clinical cohort presented mild clinical features. Hence, 46\% were not receiving treatment with immunosuppressors or immunomodulators. Ongoing treatment included nonsteroidal antiinflammatory drugs (NSAID; 53\%), corticosteroids (41\%), antimalarial drugs (39\%), and azathioprine (1\%).

\section{Discussion}

Differentiated CTD are characterized by a wide range of signs and symptoms that vary from patient to patient and in

Table 4 UCTD clinical and serological manifestations/systemic sclerosis (SSc)-like subgroup

\begin{tabular}{lccl}
\hline & $\begin{array}{l}\text { Non SSc-like } \\
\text { subgroup; } \\
N=175(\%)\end{array}$ & $\begin{array}{l}\text { SSc-like } \\
\text { subgroup; } \\
N=9(\%)\end{array}$ & $p$ \\
\hline Arthralgia & 57 & 33 & n.s. \\
Raynaud & 27 & 89 & $<0.0001$ \\
Telangiectasia & 2 & 22 & 0.012 \\
Arthritis & 34 & 0 & n.s. \\
Leukopenia & 18 & 22 & n.s. \\
Anemia & 15 & 0 & n.s. \\
Thrombocytopenia & 10 & 0 & n.s. \\
Sicca syndrome & 29 & 33 & n.s. \\
Photosensitivity & 16 & 11 & n.s. \\
Serositis & 2 & 0 & n.s. \\
Malar rash & 7 & 11 & n.s. \\
Oral ulcers & 14 & 0 & n.s. \\
Positive ANA & 100 & 100 & - \\
\hline
\end{tabular}

Table 5 UCTD clinical and serological manifestations/Sjögren (SS)like subgroup

\begin{tabular}{lccl}
\hline & $\begin{array}{l}\text { Non SS-like } \\
\text { subgroup; } \\
N=133(\%)\end{array}$ & $\begin{array}{l}\text { SS-like } \\
\text { subgroup; } \\
N=51(\%)\end{array}$ & $p$ \\
\hline Arthralgia & 60 & 69 & n.s. \\
Raynaud & 33 & 22 & n.s. \\
Arthritis & 36 & 29 & n.s. \\
Leukopenia & 15 & 33 & 0.006 \\
Anemia & 16 & 14 & n.s. \\
Thrombocytopenia & 9 & 12 & n.s. \\
Sicca syndrome & 28 & 47 & 0.027 \\
Photosensitivity & 16 & 20 & n.s. \\
Serositis & 2 & 0 & n.s. \\
Malar rash & 10 & 2 & n.s. \\
Oral ulcers & 16 & 8 & n.s. \\
Positive ANA & 100 & 100 & - \\
\hline
\end{tabular}

each patient through time. As few clinical manifestations are disease specific, there is a certain degree of overlapping between the different conditions. Classification criteria defined for each CTD permit us to identify reasonably homogenous cohorts of patients. This is rather important in pathogenesis research and clinical trials. Nevertheless, it is common for patients to present signs and symptoms suggesting CTD, but not fulfilling classification criteria for a definite disease, which are currently classified as UCTD.

The history of undifferentiated disease dates back to 1980 when LeRoy et al. proposed the concept of "undifferentiated connective tissue syndromes". Throughout the subsequent 28 years, undifferentiated CTDs have been variably defined [11]. Some cohort studies have been published, but use of variable UCTD inclusion criteria limits the possibility for comparison between cohorts and raises difficulties in the extrapolation of relating results as prognosis is concerned.

This study presents a large cross-sectional cohort of patients with UCTD and the largest one using positive ANA as inclusion criteria. We required the presence of ANA, aiming to increase the specificity for an autoimmune disorder, given the role of ANA in the pathogenesis of most of the defined CTD. In the larger published UCTD cohorts, those by Alarcon et al. [12] and Bodolay et al. [13], only 117 (55\% of 213$)$ and 166 (25\% of 665$)$ patients, respectively, presented positive ANA (Table 6). Furthermore, participating rheumatologists only requested ANA testing if there was a clinical suspicion of CTD. Indiscriminate testing for ANA in patients with musculoskeletal pain could lead to many false-positive diagnosis of UCTD, as the frequency of positive ANA in the general population is up to $8 \%$ [14]. Of notice, other rheumatologic conditions 
Table 6 UCTD clinical and serological manifestations

\begin{tabular}{|c|c|c|c|c|c|c|c|}
\hline & $\begin{array}{l}\text { Alarcon [12]; } \\
N=213(\%)\end{array}$ & $\begin{array}{l}\text { Cleg et al. [26]; } \\
N=115(\%)\end{array}$ & $\begin{array}{l}\text { Mosca [15]; } \\
N=91(\%)\end{array}$ & $\begin{array}{l}\text { Dijkstra [18]; } \\
N=22(\%)\end{array}$ & $\begin{array}{l}\text { Danieli [17]; } \\
N=165(\%)\end{array}$ & $\begin{array}{l}\text { Bodolay [13]; } \\
N=665(\%)\end{array}$ & $\begin{array}{l}\text { C. C. Vaz; } \\
N=184(\%)\end{array}$ \\
\hline Arthralgia & - & - & 80 & 77 & 58 & 50 & 66 \\
\hline Raynaud & 46 & - & 46 & 45 & 78 & 60 & 30 \\
\hline Arthritis & 70 & - & 37 & 14 & 38 & 30 & 32 \\
\hline Leukopenia & 11 & - & 41 & - & - & - & 19 \\
\hline Anemia & 23 & - & 16 & - & - & 30 & 15 \\
\hline Thrombocytopenia & 2 & - & 13 & - & 4 & 11 & 9 \\
\hline Xerophthalmia & 7 & - & 41 & 18 & 20 & 25 & 30 \\
\hline Xerostomia & 7 & - & 36 & 18 & 20 & 25 & 30 \\
\hline Photosensitivity & 10 & - & 30 & - & 22 & 23 & 17 \\
\hline Serositis & 10 & - & 5 & - & - & - & 2 \\
\hline Malar rash & 11 & - & 6 & - & - & - & 8 \\
\hline Oral ulcers & 12 & - & 4 & - & - & - & 14 \\
\hline Positive ANA & 55 & 59 & 100 & 100 & 58 & 25 & 100 \\
\hline Anti-dsDNA & - & 5 & 19 & - & 5 & - & 14 \\
\hline Anti-SSA & - & 12 & 30 & - & 14 & - & 20 \\
\hline Anti-SSB & - & 3 & 5 & - & 3 & - & 7 \\
\hline Anti-Sm & - & 3 & 1 & - & $-{ }^{\mathrm{a}}$ & - & 2 \\
\hline Anti-RNP & - & 14 & 28 & - & 7 & - & 6 \\
\hline Anti-Scl 70 & - & 0 & - & - & $-{ }^{\mathrm{a}}$ & - & 1 \\
\hline Anticentromere & - & - & - & - & $-{ }^{\mathrm{a}}$ & - & 4 \\
\hline Antihistones & - & - & - & - & $-{ }^{\mathrm{a}}$ & - & 4 \\
\hline
\end{tabular}

- not reported

${ }^{a}$ Positive ENA (unspecified) 27\%

that could justify joint pain, including fibromyalgia, were exclusion criteria for this study.

In this study, we included patients with early UCTD (less than 3 years) as well as patients with established UCTD (at least 3 years duration). Established UCTD possibly represents a cohort of patients with a more stable clinical phenotype, which is unlikely to go into remission or evolve to a differentiated CTD. Early UCTD patients potentially have a more clinically unstable disease, with a higher risk of evolving to a differentiated CTD [11].

In spite of the differences in patients' inclusion criteria between studies, epidemiological clinical, laboratorial, and immunological features observed in the present study are quite similar to those obtained in other UCTD cohorts (Table 6). Therapeutics used in patients belonging to the present clinical cohort is also similar to those described in previous studies.

A fundamental question to consider is how to estimate the prognosis of UCTD, particularly whether the clinical situation will evolve to a defined CTD and the possibility of foreseeing when this might occur. The published prospective studies [12-18] found an evolving frequency that varies from $5 \%$ to $68 \%$ of patients. According to this data, it is estimated that $20-40 \%$ of patients with early UCTD will develop a definite CTD [11]. Development to differentiated CTD might occur from months to years after the first UCTD manifestations. Progression risk seems to be higher during the disease's first 5 years $[11,19]$.

UCTD might evolve to any of the different defined CTD. Nevertheless, some clinical and laboratorial features may suggest a higher probability of disease evolution to occur to a certain CTD instead of others. In the present clinical cohort, we found that patients with anti-dsDNA/ anti-Sm, anticentromere/anti-Scl70, or positive anti-SSA/ anti-SSB more frequently present a set of manifestations close to SLE, SSc, or SS, respectively.

In our UCTD cohort, $40 \%$ presented a lupus-like disease. In the cohort assessed by Swaak and Smeenk [20], the positivity of anti-dsDNA antibodies was predictive for evolution to SLE. Calvo-Álen et al. [21] noted that alopecia, serositis, positive Coombs test, anti-dsDNA, antiSm, positive test for syphilis, and ANA with homogenous pattern correlated significantly to differentiation toward SLE. Danieli et al. [16, 17] identified fever, anticardiolipin antibodies, and anti-dsDNA as predictive factors for SLE. Vila et al. [22] demonstrated that malar rash, oral ulcerations, 
anti-dsDNA positivity, and decreased C4 were statistically significant in predicting the evolution to SLE. Cavazzana et al. [23] noticed that leukopenia was more frequently detected in patients who developed a definite CTD and that anti-dsDNA was predictive of evolution to SLE.

Also in the study of Danieli et al. [16, 17], sicca symptoms, Raynaud's phenomenon, sclerodactylia, esophageal dysfunction, and ANA with nucleolar pattern as predictive factors for the development of systemic sclerosis and Raynaud's phenomenon, xerostomia, and anti-SSA as predicative factors for Sjögren syndrome. In our UCTD cohort, patients with anticentromere antibodies or anti-Scl 70 presented systemic sclerosis-like clinical features, and the ones with anti-SSA and/or anti-SSB antibodies presented a Sjögren syndrome-like disease.

In our cohort, most patients had a very limited profile of ANA specificities, with no detectable antigen specificity in $61 \%$ of patients and just one in another $26 \%$. Mosca et al. [1] reported a correlation between SLE development and the accumulation of autoantibodies. Furthermore, the analysis of the autoantibodies specificities during followup showed that UCTD patients with a stable profile do not develop new specificities. On the contrary, patients developing SLE develop new antibody specificities during the course of the follow-up [11]. In general, autoantibodies might be detectable several years before the onset of symptoms. In a study on 130 SLE patients, at least one antibody was present in $88 \%$ of patients before the diagnosis [24].

The best way to classify the cases with features close to a definite CTD, like SLE, SSc, or SS, remains a matter of debate. These may be seen either as UCTD or alternatively as incomplete forms of a defined CTD. In an attempt to distinguish "true" UCTD from incomplete CTD, there has been suggested a preliminary set of exclusion criteria in addition to classification criteria for UCTD [25]. These included clinical manifestations and ANA reactivities considered more specific for a definite CTD. According to this view, incomplete forms of defined CTD would belong to the same spectra of the related CTD and not to UCTD. However, there are no clinical or immunological features truly specific of a defined CTD. Excluded from the UCTD group, the frontiers of incomplete forms of defined CTD would remain very difficult to establish, and in practice, a group of unclassifiable cases, neither UCTD nor defined CTD, would remain.

Our study has some limitations. It is a cross-sectional evaluation of patients with variable disease duration, and those with UCTD at presentation who developed features of a differentiated CTD before the time of this evaluation were not included. Also, the presence of "disease specific" antibodies may have taken the clinician to valuate some manifestations in detriment of others, constituting a potential source of bias for the definition of UCTD subgroups.
In summary, in this study, we describe a large cohort of UCTD patients and found that more than half of the patients present a SLE-, SS-, or SSc-like disease. This and previous studies of UCTD partake in predominantly mild clinical profile and need for mostly symptomatic therapy. According to available data, many patients with UCTD have a favorable prognosis. However, we find it advisable for these patients to be regularly followed up, even if only presenting with benign manifestations, so as to detect eventual evolution to a more severe specific CTD. There is a need to establish better classification criteria and of longterm observational study of UCTD cohorts to better understand this entity and its prognosis and development of trials of promising drugs for preventing disease progression.

Disclosures None.

\section{References}

1. Mosca M, Neri R, Bombardieri S (1999) Undifferentiated connective tissue diseases: a review of the literature and a proposal for preliminary classification criteria. Clin Exp Rheumatol 17(5):615-620

2. Zold E, Szodoray P, Gaal J, Kappelmayer J, Csathy L, Gyimesi E, Zeher M, Szegedi G, Bodolay E (2008) Vitamin D deficiency in undifferentiated connective tissue disease. Arthritis Res Ther 10 (5):R123

3. James JA, Kim-Howard XR, Bruner BF, Jonsson MK, McClain MT, Arbuckle MR, Walker C, Dennis GJ, Merrill JT, Harley JB (2007) Hydroxychloroquine sulfate treatment is associated with later onset of systemic lupus erythematosus. Lupus 16(6):401409

4. Hochberg MC (1997) Updating the American College of Rheumatology revised criteria for the classification of systemic lupus erythematosus. Arthritis Rheum 40:1725

5. Petri M (2005) Review of classification criteria for systemic lupus erythematosus. Rheum Dis Clin North Am 31(2):245-254 vi

6. Subcommittee for Scleroderma Criteria of the American Rheumatism Association Diagnostic and Therapeutic Criterion Committee (1980) Preliminary criteria for the classification of systemic scleroris (scleroderma). Arthritis Rheum 23:581-590

7. LeRoy EC, Medsger TA (2001) Criteria for the classification of early systemic sclerosis. J Rheumatol 28:1573-1576

8. Johnson SR, Feldman BM, Hawker GA (2007) Classification criteria for systemic sclerosis subsets. J Rheumatol 34(9):18551863

9. Vitali C, Bombardieri S, Moutsopoulos HM, Balestrieri G, Bencivelli W, Bernstein RM, Bjerrum KB, Braga S, Coll J, de Vita $S$ et al (1993) Preliminary criteria for the classification of Sjögren's syndrome. Results of a prospective concerted action supported by the European community. Arthritis Rheum 36:340347

10. Vitali C, Bombardieri S, Jonsson R, Moutsopoulos HM, Alexander EL, Carsons SE, Daniels TE, Fox PC, Fox RI, Kassan SS, Pillemer SR, Talal N, Weisman MH (2002) European Study Group on Classification Criteria for Sjögren's Syndrome. Classification criteria for Sjögren's syndrome: a revised version of the European criteria proposed by the American-European Consensus Group. Ann Rheum Dis 61(6):554-558 
11. Mosca M, Tani C, Bombardieri S (2007) Undifferentiated connective tissue diseases: a new frontier in rheumatology. Best Pract Res Clin Rheumatol 21(6):1011-1023

12. Alarcon GS, Williams GV, Singer JZ, Steen VD, Clegg DO, Paulus HE, Billingsley LM, Luggen ME, Polisson RP, Willkens RF et al (1991) Early undifferentiated connective tissue disease. I Early clinical manifestations in a large cohort of patients with undifferentiated connective tissue diseases compared with cohorts of well established connective tissue disease. J Rheumatol 18:1332-1339

13. Bodolay E, Csiki Z, Szekanecz Z, Ben T, Kiss E, Zeher M, Szücs G, Dankó K, Szegedi G (2003) Five-year follow-up of 665 Hungarian patients with undifferentiated connective tissue disease. Clin Exp Rheumatol 21:313-320

14. Mosca M, Neri R, Bencivelli W, Tavoni A, Bombardieri S (2002) Undifferentiated connective tissue disease: analysis of 83 patients with a minimum follow up of 5 years. J Rheumatol 29:2345-2348

15. Mosca M, Tavoni A, Neri R, Bencivelli W, Bombardieri S (1998) Undifferentiated connective tissue diseases: the clinical and serological profiles in 91 patients followed for at least 1 year. Lupus 7:95-100

16. Danieli MG, Fraticelli P, Salvi A, Gabrielli A, Danieli G (1998) Undifferentiated connective tissue diseases: natural history and evolution into definite CTD assessed in 84 patients initially diagnosed as early UCTD. Clin Rheumatol 17:195-201

17. Danieli MG, Fraticelli P, Franceschini F, Cattaneo R, Farsi A, Passaleva A, Pietrogrande M, Invernizzi F, Vanoli M, Scorza R, Sabbadini MG, Gerli R, Corvetta A, Farina G, Salsano F, Priori R, Valesini G, Danieli G (1999) Five-year follow-up of 165 Italian patients with undifferentiated connective tissue diseases. Clin Exp Rheumatol 17:585-591

18. Dijkstra S, Nieuwenhuys EJ, Swaak AJ (1999) The prognosis and outcome of patients referred to an outpatient clinic for rheumatic diseases characterized by the presence of antinuclear antibodies (ANA). Scand J Rheumatol 28:33-37

19. Mosca M, Tani C, Bombardieri S (2008) A case of undifferentiated connective tissue disease: is it a distinct clinical entity? Nat Clin Pract Rheumatol 4(6):328-332
20. Swaak AJ, van de Brink H, Smeenk RJ, Manger K, Kalden JR, Tosi S, Marchesoni A, Domljan Z, Rozman B, Logar D, Pokorny G, Kovacs L, Kovacs A, Vlachoyiannopoulos PG, Moutsopoulos HM, Chwalinska-Sadowska H, Dratwianka B, Kiss E, Cikes N, Anic B, Schneider M, Fischer R, Bombardieri S, Mosca M, Graninger W, Smolen JS (2001) Study group on incomplete SLE and SLE with disease duration longer than 10 years. Incomplete lupus erythematosus: results of a multicenter study under the supervision of the EULAR Standing Commitee on International Clinical Studies Including Therapeutic Trials (ESCISIT). Rheumatol 40:89-94

21. Calvo-Alen J, Alarcon GS, Burgard SL, Burst N, Bartolucci AA, Williams HJ (1996) Systemic lupus erythematosus: predictors of its occurrence among a cohort of patients with early undifferentiated connective tissue disease: multivariate analyzes and identification of risk factors. J Rheumatol 23:469-475

22. Vila LM, Mayor AM, Valentín AH, García-Soberal M, Vilá S (2000) Clinical outcome and predictors of disease evolution in patients with incomplete lupus erythematosus. Lupus 9:110 115

23. Cavazzana I, Franceschini F, Belfiore N, Quinzanini M, Caporali $\mathrm{R}$, Calzavara-Pinton $\mathrm{P}$, Bettoni L, Brucato A, Cattaneo R, Montecucco C (2001) Undifferentiated connective tissue disease with antibodies to Ro/SSA: clinical features and follow up of 148 patients. Clin Exp Rheumatol 19:403-409

24. Arbuckle MR, McClain MT, Rubertone MV, Scofield RH, Dennis GJ, James JA, Harley JB (2003) Development of autoantibodies before the clinical onset on systemic lupus erythematosus. N Eng J Med 349(16):1526-1533

25. Doria A, Mosca M, Gambari PF, Bombardieri S (2005) Defining unclassifiable connective tissue diseases: incomplete, undifferentiated, or both? J Rheumatol 32:213-215

26. Clegg DO, Williams HJ, Singer JZ, Steen VD, Schlegel S, Ziminski C, Alarcón GS, Luggen ME, Polisson RP, Willkens RF et al (1991) Early undifferentiated connective tissue disease. II. The frequency of circulating antinuclear antobodies in patients with early rheumatic diseases. J Rheumatol 18:1340-1343 